\title{
Commentaries
}

\section{Commentaries on the End of Year Editorial on the definition of ischaemia in last December's issue}

\section{From A G Kléber}

The articles by Ingwall ${ }^{1}$ and Verdonck ${ }^{2}$ discuss the important issue and the partially controversial results of measurements of intracellular sodium activity during acute myocardial ischaemia. Several arguments have been raised by both authors to explain the apparent discrepancies between intracellular sodium measurements performed either using NMR techniques or ion-sensitive microelectrodes (ISME): as stated by both authors, potential inaccuracies or pitfalls with either method and/or species differences might partially explain the discrepancies in experimental results.

Methodological problems indeed may play a role in the quantitative interpretation of ionic levels and fluxes, and they are likely to be inherent to both methods. ISME measure ion activities in surface cells of ischaemic tissue. Calibration of ISME is made in solutions with either $\mathrm{Cl}^{-}$or a mixture of $\mathrm{Cl}^{-}$and $\mathrm{HCO}_{3}^{-}$as an anionic background and it is not clear how the complex intracellular "jelly", the change in osmolality and ischaemic metabolites may affect the relationship between the concentration and the activity (which is measured by ISME) of a test ion. With NMR, the calculated ion concentrations depend, as mentioned by Ingwall, on the accuracy of determination of the intra- to extracellular space ratio and the NMR visible ion fraction in test and ischaemic conditions. Moreover NMR measurements during ischaemia, in most instances, are performed in whole hearts, and represent measurements of average ionic changes. Therefore they cannot account for the inhomogeneities in intracellular and extracellular ionic changes in regional and global ischaemia, as discussed below.

Both articles argue about the pathophysiological background of changes in $\mathrm{Na}^{+}$, during ischaemia in very much the same way: $\mathrm{Na}_{i}^{+}$will eventually be the result of a balance between the unidirectional transmembrane fluxes of sodium, and the crucial question is "how and to what extent do the various components of sodium efflux and influx change during ischaemia?" Besides the fact that most of these components cannot be assessed separately during ischaemia in vivo, the answer to this question is complicated by two other considerations.

First, acute ischaemia is characterised by distinct phases or periods, and it is well known that the expression and duration of these periods vary with species. A first phase of reversible ischaemia (during which most of the "malign" arrhythmias occur, and during which ISME measurements are possible) is characterised by (1) acute electrical changes, associated with accumulation of extracellular potassium, (2) normal electrical and metabolic cell to cell coupling, (3) a rapid decrease of active tension development in presence of normal resting tension (no ischaemic contracture), and (4) only a moderate decrease of cellular [ATP] and of the energetic driving force, that is, the cytosolic free energy change of ATP hydrolysis. The change of $\mathrm{pH}_{\mathrm{i}}$ and $\left[\mathrm{Ca}^{2+}\right]$ during this phase is controversial. In blood perfused hearts, the intracellular acidification is relatively small, much less than in crystalloid perfused hearts. The second phase of ischaemia starts abruptly with an almost simultaneous development of ischaemic contracture, electrical cell to cell uncoupling, and breakdown of ionic homeostasis. In large animals, such as dog and pig and probably also in man, the duration of this first phase is relatively long (15-20 min) whereas it is very short in rat hearts (4-5 min) with only a small $\left[\mathrm{K}^{+}\right]_{0}$ accumulation. This may partially explain why during the first 15 minutes after coronary occlusion, a large increase in intracellular sodium was demonstrated using NMR in rat hearts whereas only relatively small increases were measured in guinea pig and rabbits with ISME.

A second consideration, which is likely to have an impact on the interpretation of $\left[\mathrm{Na}^{+}\right]_{\mathrm{i}}$ measurements, relates to the fact that ionic changes in ischaemia are non-homogeneous Large gradients of $\left[\mathrm{K}^{+}\right]$and $\mathrm{pH}$ have been measured between the centre and the border zone in regional ischaemia, and such gradients are likely to be present in global ischaemia as well. They have been partially attributed to diffusion of $\left[\mathrm{K}^{+}\right]$ and $\left[\mathrm{H}^{+}\right]$into the normoxic zone or into surrounding fluid compartments, and partially by diffusion of $\mathrm{CO}_{2}$ formed from bicarhonate by ischaemic metabolism. Several reports have shown that the change in $\left[\mathrm{Na}^{+}\right]_{\text {in }}$ depend on the inhomogeneity in $\left[\mathrm{K}^{+}\right]$and $\left[\mathrm{H}^{+}\right]$. In ischaemic cells of guinea pig heart, which are depolarised to about $-50 \mathrm{mV}$ (in presence of $18-20 \mathrm{mM}\left[\mathrm{K}^{+}\right]_{\mathrm{o}}$ ), no significant increase in $\left[\mathrm{Na}^{+}\right]_{\mathrm{i}}$ was measured with ISME, whereas an increase by about $6 \mathrm{mM}$ during early ischaemia was measured in moderately depolarised ischaemic rabbit hearts $\left(\left[\mathrm{K}^{+}\right]_{0}=\right.$ 10-12 mM). This confirmed earlier measurements with ISME in simulated ischaemia indicating that $\left[\mathrm{Na}^{+}\right]_{j}$ in ischaemia is critically dependent on sodium influx, which is smaller in depolarised non-excitable cells than in moderately depolarised cells which still produce action potentials. Taking all these measurements in a unifying concept would suggest that $\left[\mathrm{Na}^{+}\right]_{\mathrm{i}}$ during the early phase of acute ischaemia can rise considerably at sites where increases in $\left[\mathrm{K}^{+}\right]$and $\left[\mathrm{H}^{+}\right]$are small but only moderately at depolarised ischaemic sites where marked changes in $\left[\mathrm{K}^{+}\right]$and $\left[\mathrm{H}^{+}\right]$are present.

$\Lambda$ further question which is often discussed in the context of ischaemia relates to the degree of the inhibition of the $\mathrm{Na}^{+}$/ $\mathrm{K}^{+}$pump and the relation to the change of the energetic driving force. It is evident that the energetic driving force for $\mathrm{Na}^{+} / \mathrm{K}^{+}$pumping decreases almost immediately after coronary occlusion. The decrease, however, is moderate in the early phase and it reaches a plateau after two to three minutes. In normoxia with normal intracellular $\left[\mathrm{Na}^{+}\right]_{i}$, the working point of the $\mathrm{Na}^{+} / \mathrm{K}^{+}$pump is far from energetic equilibrium. This means that there is a large cnergetic rescrve resulting in a match of sodium pumping with sodium influx if the sodium load is increased, at the expense of only a moderately increase in $\left[\mathrm{Na}^{+}\right]_{\mathrm{i}}$. Even if an inhibition of $30 \%$ of $\mathrm{Na}^{+} / \mathrm{K}^{+}$pumping (inhibition of $\mathrm{V}_{\max }$ ) during acute ischaemia is postulated, the calculated increase in $\left[\mathrm{Na}^{+}\right]_{\mathrm{i}}$ will be relatively small $(4-8 \mathrm{mM})$ if the sodium influx (so called cellular sodium load) remains 
unchanged. In this case, decreasing sodium influx will result even in a smaller increase in $\left[\mathrm{Na}^{+}\right]_{i}$, increasing sodium influx will result in a larger $\left[\mathrm{Na}^{+}\right]_{i}$. Therefore, the question of the change in sodium influx during acute ischaemia is crucial for the understanding of sodium homeostasis. Although depolarisation per se and development of inexcitability decrease the cellular sodium load, $\mathrm{Na}^{+} / \mathrm{H}^{+}$exchange and probably other ionic transfer mechanisms in ischaemia increase sodium influx. The amount of this increase depends on the changes in intracellular and extracellular $\mathrm{pH}$, which in turn depend markedly on the perfusion conditions and are nonhomogeneous, as discussed by Verecke.

One more general but speculative consideration may, however, indicate that sodium influx as a whole is decreased during acute ischaemia: [ATP], $P_{i}$, and the free energy change for ATP hydrolysis stabilise during acute ischaemia (for a brief period) after the initial change. Since energy production is markedly decreased during ischaemia, a transiently stabilised energy driving force implicates a rundown of energy consuming processes as well. This has been shown for the decrease in active tension development, cellular calcium cycling and ionic loads associated with electrical excitation. A large sodium influx during the acute phase of ischaemia would be expected to increase energy consumption, to disturb the energetic steady state, and to lead to a breakdown of homeostasis via positive feedback. Such a process may be responsible for the transition to the second phase of ischaemia but it is unlikely to happen during the first peracute phase, because of the observed transient stability. The concept that "acute hibernation", that is, a decrease of energy consuming processes in general (as a regulatory change), rather than a continuous decrease in the energy driving force, is the key event in peracute ischaemia is also supported by the observation that both a contractile reserve and a reserve in $\mathrm{Na}^{+} / \mathrm{K}^{+}$pumping are maintained in the first minutes after interruption of coronary flow.

ANDRÉ G KLÉBER

Department of Physiology, University of Bern, Bern, Switzerland.

1 Ingwall JS. How high does intracellular sodium rise during acute myocardial ischaemia? A view from NMR spectroscopy. Cardiovasc Res 1995;29:000.

2 Verdonck F. How high does intracellular sodium rise during acute myocardial ischaemia? Cardiovasc Res 1995;29:000.

\section{From M Tani}

An increase in the intracellular sodium concentration $\left(\left[\mathrm{Na}^{+}\right]_{i}\right)$ during ischaemia and reperfusion is thought to play an important role in myocardial injury because it is followed by excess calcium influx via the $\mathrm{Na}^{+} / \mathrm{Ca}^{2+}$ exchanger. ${ }^{1}$ The calcium overload results in activation of calcium dependent adenosine triphosphatases (ATPases), lipases, and proteases, as well as in mitochondrial dysfunction, muscle contracture, and disruption of the sarcolemma and mitochondrial membranes. However, apparently the data on how much the $\left[\mathrm{Na}^{+}\right]_{\mathrm{i}}$ actually increases have been contradictory.

As Ingwall states, the $\left[\mathrm{Na}^{+}\right]_{\mathrm{i}}$ depends on the rates of sodium influx and efflux. ${ }^{2}$ The pathways for sodium influx include sodium channels, the $\mathrm{Na}^{+} / \mathrm{H}^{+}$exchanger, the $\mathrm{Na}^{+} / \mathrm{Ca}^{2+}$ exchanger, the $\mathrm{K}^{+} / \mathrm{Na}^{+} / 2 \mathrm{Cl}^{-}$cotransporter, the $\mathrm{Na}^{+} / \mathrm{HCO}_{3}$ cotransporter, and other cotransporter systems, whereas the pathways for sodium efflux are the $\mathrm{Na}^{+} / \mathrm{K}^{+}$-ATPase and the $\mathrm{Na}^{+} / \mathrm{Ca}^{2+}$ exchanger (reverse mode). These mediators of sodium flux are regulated by many factors including membrane potential or action potential, intraccllular or extraccllular ion concentrations and transsarcolemmal ion concentration gradients, ATP, ADP, inorganic phosphate, or other metabolites, and $\mathrm{pH}$. Many of these factors are interrelated. Their relative contributions to net sodium flux during ischaemia are thus determined by the experimental conditions; regulatory factors are affected by the rate of coronary flow, duration of ischaemia, level of myocardial energy demand, whether heart is arrested or beating (paced), the capacity for extracellular diffusion, and the type of perfusate.

Although the discrepancy in measured $\left[\mathrm{Na}^{+}\right]_{i}$ values during ischaemia appears to depend on the techniques [ion selective electrode (ISE) versus nuclear magnetic resonance (NMR)] used for determination of $\left[\mathrm{Na}^{+}\right]_{i}$, differences also exist in the experimental protocols, especially with regard to restriction of ischaemic conditions by the limitations of the technique applied.

As Verdonck pointed out, it is very hard to measure $\left[\mathrm{Na}^{+}\right]$ in the endocardium of a beating heart over relatively long periods of time with the ISE technique. ${ }^{3}$ Because of these limitations, the ISE techniquc provides data from epicardial cells that are subjected to relatively mild ischaemia, even when the heart is globally ischaemic. ${ }^{4-8}$ Because intracellular acidification or the transsarcolemmal $\mathrm{H}^{+}$gradient is less pronounced in regions of mild ischaemia, both the inhibition of the $\mathrm{Na}^{+} / \mathrm{K}^{+}$-ATPase and the activation of the $\mathrm{Na}^{+} / \mathrm{H}^{+}$ exchanger are less prominent and the production of ATP continues, resulting in the preservation of $\mathrm{Na}^{+} / \mathrm{K}^{+}$-ATPase activity during the later period of ischaemia. The scenario is supported by the observation of regional differences in $\left[\mathrm{Ca}^{2+}\right]_{i}$ in the globally ischaemic heart. ${ }^{9}$ Recently, Allen reported that $\left[\mathrm{Na}^{+}\right]_{1}$ increases relatively slowly during global ischaemia in the epicardial layers of myocytes of rat heart when $\left[\mathrm{Na}^{+}\right]_{\mathrm{i}}$ was measured with the technique with a novel fluorescent indicator SBFI. ${ }^{10}$

In contrast, NMR allows the measurement of $\left[\mathrm{Na}^{+}\right]_{\mathrm{i}}$ in ischaemic/reperfused beating hearts. However, this technique also has limitations and requires the preadministration of shift reagents to discriminate between intracellular and extracellular sodium. ${ }^{2}$ Shift reagents are charged anionic complexes of lanthanides and chelate various cations, including calcium, which change the equilibrium between free and bound ions. The spatial or temporal resolution of NMR is not sufficient for regional or real time analysis of $\left[\mathrm{Na}^{+}\right]_{i}$. The technique therefore provides only time averaged values of several beats in a relatively large region of interest or in the whole heart of small mammals. ${ }^{11-15}$ The visibility of intracellular sodium signals by NMR can be affected by changes in the intracellular concentrations of $\mathrm{H}^{+}$or cations, which can decrease the number of anionic binding sites for sodium.

Rapid pacing of rat hearts results in a decrease in left ventricular systolic pressure (negative staircase), whereas rapid pacing increases left ventricular systolic pressure in many other small mammals, including guinea pigs and rabbits. The sensitivity of the rat heart to cardiac glycosides also differs from that of hearts of many other small mammals. ${ }^{16}$ Ingwall points out that marked species differences also are apparent for the relationship between the rate of sodium accumulation and changes in the concentrations of ATP and its hydrolysis products in small mammalian hearts. In addition, slow beating hearts of larger mammals, such as pigs, show slower rates of high energy phosphate depletion during ischaemia compared with the fast beating hearts of small mammals. ${ }^{17}$ These observations indicate that the relative contributions of the various mediators of sodium flux vary among species. 